\title{
IMPACTS AND FLUID-STRUCTURE INTERACTION IN PRESSURIZED WATER REACTOR SAFETY ANALYSIS *
}

\author{
Ulrich SCHUMANN \\ Kernforschungszentrum Karlsruhe, Institut für Reaktorentwicklung, Projekt Nukleare Sicherheit, Postfach 3640, D.7500 Karlsruhe, \\ Fed. Rep. Germany
}

\begin{abstract}
A numerical method is described for the analysis of coupled three-dimensional fluid-structure motion with impacts between structural parts at rigid or flexible supports with small clearances. The method is used for the analysis of the blowdown loadings and the response of internal structures in the vessel of a pressurized water reactor (PWR) in the hypothetical event of a sudden break of a coolant inlet pipe. The method is a generalization of the existing code FLUX which simulates the three-dimensional fluid-structure motion by means of an implicit time integration scheme. The additional supports with clearances are taken into account by applying support forces to the freely moving fluid-structure system. The forces are determined such that the kinematic constraints are enforced at each time step. Numerically, these forces are determined efficiently using a precomputed influence matrix which defines the dynamic displacement per time step at each support due to a unit force at each other support. According to the actually "active" supports the relevant influence matrix in constructed. Energy is conserved for rigid supports and for supports which are so flexible that the impact time is large in comparison to the time steps. Treatment of plastic supports is possible.

An application of the new method is demonstrated by analysis of the core barrel motion in a PWR with and without impacts at the lower core barrel edge and at the upper flange. The results show the large effects of such impacts in changing the global motions. Large local impact forces and accelerations appear. The interaction with the fluid reduces these loads. By proper design of the supports the resultant stresses can be minimized. Thus the method can be used to demonstrate and enlarge nuclear reactor safety.
\end{abstract}

\section{Introduction}

In the safety analysis of a pressurized water reactor (PWR) with respect to the vessel internal structures, several accident conditions are postulated. Among these assumed accidents, the blowdown caused by a sudden complete break of a primary coolant pipe plays a dominant role as it is the most severe case with respect to the loads applied to the components. During the last few years several computer codes have been developed for analysis of the two- or three-dimensional coupled fluid-structure motions in a PWR under such conditions $[1,2]$. The current research concentrates on the analysis of the core barrel shown in fig. 1 . The HDR blowdown experiments [3] constitute a mile-stone on this road of research. A first series of tests has been performed in 1980 [4]. By parametric studies and comparison to these experiments it has been shown that a

* This paper was the winner of the SMiRT-6 Jaeger prize. It was selected by a technical review committee for its technical merit among a group of 14 papers that entered the contest for the Jaeger prize. realistic analysis requires very fine spatial resolution and simultaneous simulation of the fluid and structure motion. Thus very time consuming computer models with many degrees of freedom (typically 5000) are necessary. Therefore one line of research follows the development of efficient algorithms in order to limit the required computer time. This is necessary in particular because nuclear reactor licensing does not only require just one run with a code rather then parametric studies in order to understand the effects and possibility to optimize the design.

In this respect, we have developed the computer code FLUX [5]. FLUX uses an implicit time integration scheme. At each time step a large system of linear equations has to be solved. This is accomplished by means of fast Fourier transform and fast elliptic solvers.

However, in the existing multi-dimensional models the core barrel has been assumed to be ideally supported without any clearances. On the other hand, in a real reactor situation, the core barrel is flexibly supported at the upper flange with finite small clearances. Springs enforce to some extent the core barrel in its reference position. At the lower end, the core barrel's 


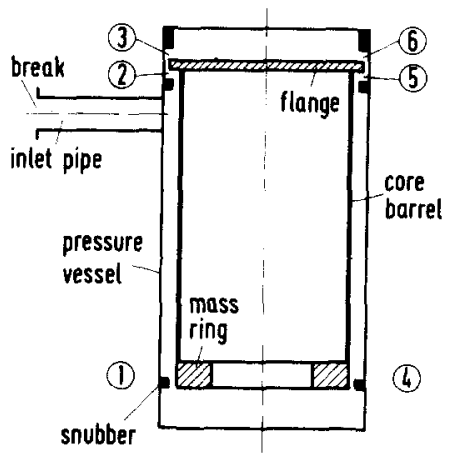

Fig. 1. Model-geometry for a pressurized water reactor vessel showing six supports with clearances for example.

radial motion is restrained by "snubbers", see fig. l. If the core barrel hits these supports than the flexibility of the snubbers as indicated in fig. 2 is an important parameter. Elastic and plastic reactions will appear. Large local load peaks are to be expected. At rigid supports the duration of the contact between the impacting structures can be very small and the forces very high although the impulse transmitted is finite. It is not clear a priori whether such constraints reduce or enlarge the deformations and stresses. In particular the role of the coupled fluid motion has not been previously investigated in case of impacts. Thus quantitative evalution of such motions is an important safety issue.

For PWR internals some previous work regarding motions with impacts has been reported [6]. For example Böhm and Lafaille [7] have performed an extensive analysis of such motions. They used a multimass system connected with springs and dashpots. The fluid motion was determined for rigid structures. The resultant loads were imposed to the structural system which includes rough estimates for the effective fluid mass. However,

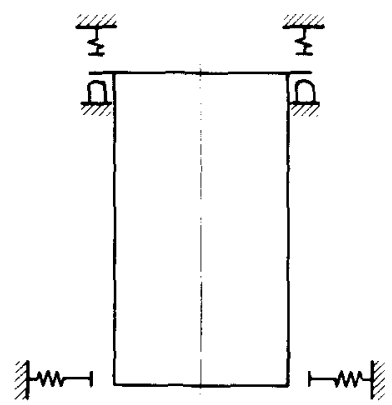

Fig. 2. Rigid and flexible supports. the mass matrix is assumed to be diagonal, which allows for explicit integration schemes, and the number of degrees of freedom is about two orders of magnitude smaller than required for realistic analysis in a three-dimensional model. Thus, an efficient method for fluidstructure analysis with impacts does not seem to have been described in the literature.

Supports with clearances make the structural system extremely nonlinear. Closure of a gap due to motion at any one of the supports causes a step change of the dynamic properties of the system. One can easily verify that for $m$ supports $2^{m}$ different support configurations are possible. Thus it might seem difficult to include such impact effects in a computer code which, like FLUX, is based on implicit time integration for efficiency purposes and which solves large linear systems of equations at each time step.

In this paper an appropriate and efficient method will be described. The method introduces support forces as additional unknowns. These forces are zero at "passive" supports with unclosed gaps. At "active" supports with closed gaps the forces are determined in a way that the kinematic constraints are enforced. For this purpose a nonlinear variant of the influence matrix technique $[5,8]$ will be introduced. The nonlinear problem with impacts is solved by solving the linear problem (using the existing solution scheme) twice at each time step and a small amount of additional computations in between.

Fig. I shows the example problem considered in this paper. Here $m=6$ supports with clearance are introduced. It is an easy matter to extend for a larger number of such supports. The method is efficient as long as $m$ is small in comparison to the total number of degrees of freedom and to the number of time steps.

We do not compete with the amount of details covered by Böhm and Lafaille [7] with respect to the reactor core. Core models for three-dimensional fluidstructure analysis are still under development [9]. In the present analysis, the mass of the core is included approximately in the form of a stiff mass ring connected rigidly to the lower core barrel edge as indicated in fig. 1.

Special attention has to be given to the energy conservation in the numerical model during impact. Our goal is to develop a scheme which is free of large numerical damping. Plastic deformations and friction forces should be added explicitly thereafter.

This will be discussed in sections 7 and 8 . In section 2 the support and impact conditions are formally defined. In section 3 the essential features of the original fluid-structure model are summarized. In section 4 the 
model including impact forces is given. Its time discretization is defined in section 5. Section 6 contains the essential part of the method, i.e. the solution algorithm for the system of equations which has to be solved at each time step. Finally, in section 9, prototype applications of the method are reported.

\section{Support and impact conditions}

Let the configuration under consideration consist of $J$ elastic substructures with number $\gamma=1,2, \ldots, J$ which can move relative to each other with clearances and possible impacts at $\mathrm{m}$ supports. For example, in fig. 1 we have the substructures $\gamma=1$, the core barrel, and $\gamma=2$, the pressure vessel, so that $J=2$. We do not exclude the possibility that the substructures are elastically connected by connections without clearances. For example, the core barrel flange is connected to the pressure vessel by springs even when all supports with clearances show positive gaps.

We assume that the maximum clearance gaps are small in comparison to characteristic lengths of the configuration (e.g., the downcomer width) and that the impact direction does not depend on the displacement field. Fluid layers which have to be squeezed aside at such supports have not been included in the analysis.

Let $\boldsymbol{w}_{\gamma}(x, t)$ be the displacement field of the substructure $\gamma$ as a function of space $\boldsymbol{x}$ and time $t$. Because of the assumed smallness of the clearances, each of the $m$ possible impact locations is to sufficient accuracy given by one position vector $\boldsymbol{x}_{i}, i=1,2, \ldots, m$. The gaps between the substructures in their reference configuration, where $\boldsymbol{w}^{\prime} \equiv 0$, are denoted by $s_{j}$. This is the shortest distance between the adjacent impact points of the two substructures in the vicinity of $x_{i}$, see fig. 3. Let $\alpha_{i}$ and $\beta_{i}$ be the numbers of the two substructures envolved in the possible impact near $x_{i}, 1 \leqslant \alpha_{i} \leqslant J, 1 \leqslant \beta_{i}<J, \alpha_{i} \neq$ $\beta_{i}$. Then we can define the vector $e_{i}$ which is the unit vector into which the substructure $\alpha_{i}$ has to move in order to hit substructure $\beta_{i}$. As stated above, we assume $e_{i}$ to be constant during the motions.

The actual gap at a given instant of time $t$ is $g_{l}(t)$ and determined by

$g_{i}:=s_{i}-e_{i}\left[\boldsymbol{w}_{\alpha_{i}}\left(\boldsymbol{x}_{i}, t\right)-\boldsymbol{w}_{\beta_{i}}\left(\boldsymbol{x}_{i}, t\right)\right], \quad i=1,2, \ldots, m$.

Thus the impact state $\theta_{i}$, which is either "active" $\left(\theta_{i}=1\right)$ or "passive" $\left(\theta_{i}=0\right)$ is determined by

$\theta_{i}=0 \leftrightarrow g_{i}>0$,

$\theta_{i}=1 \leftrightarrow g_{i} \leqslant 0$.

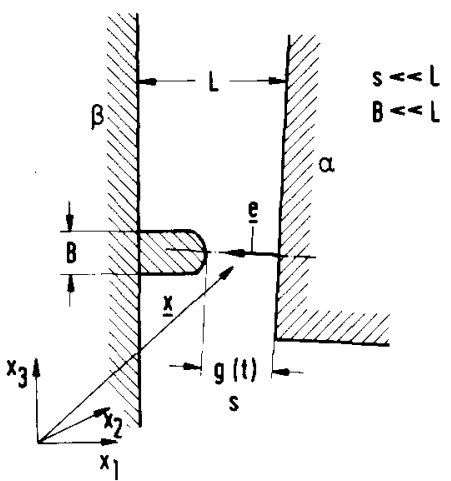

Fig. 3. Support notation.

The actual number of active impacts, $m^{\prime}=m^{\prime}(t)$, is given by

$m^{\prime}:=\sum_{i=1}^{m} \theta_{i}$

We will admit "negative gaps" $g_{i}$ for flexible supports. In this case $\left|g_{i}\right|$ is the amount of compression of the spring which represents the support.

For flexible supports we assume that the force $k_{i}$ reacting opposite to $e_{\mathrm{i}}$ is given by

$$
\begin{array}{ll}
F_{i} k_{i}=g_{i} & \text { for } \theta_{i}=1, \\
k_{i}=0 & \text { for } \theta_{i}=0 .
\end{array}
$$

Here the flexibility of the support is characterized by the inverse stiffness $F_{i} \geqslant 0$. For rigid supports $F_{i}=0$. In this case, eq. (5) requires $g_{i}=0$ and leaves the impact force $k_{i}$ yet undetermined. Because of eqs. (3) and (5) we see that the impact forces are non-positive,

$k_{i} \leqslant 0$.

On can envision several generalizations of the above definitions. For example we could consider flexibilities depending on $g_{i}$ and its time derivatives, impacts which transmit forces perpendicular to $e_{i}$ or impacts which are due to angular motion constraints and which imply impact moments instead of forces. If necessary, corresponding extensions can be introduced.

\section{The fluid-structure model without impacts}

The code FLUX simulates the three-dimensional fluid motion including fluid-structure interaction. The fluid is modeled as compressible potential flow with 
constant speed of sound. The core barrel motion is approximated by a shell model. At present, we can choose among the models CYLDY2 [10] and CYLDY3 [11] where the latter is more accurate while the former is simpler to use. Both solve Flügge's shell equations. The coupling scheme imposes the pressure loads on the structure and insures continuity of normal velocity and acceleration at the undeformed fluid-structure interface. The fluid equations are discretized by finite differences in space. The implicit Newmark- $\beta$-scheme [12] is used for time integration with respect to pressure and shell deflection. Fluid convection is treated explicitly. Fluid and structural damping can be included by parametric models. At the break the discharge pressure is prescribed by an input function. Models for the vessel and core dynamics coupled to the fluid and core barrel are under preparation.

The structural deflections $\boldsymbol{w}_{\gamma}(x, t)$ of the substructures $\gamma(\gamma=1,2, \ldots, J)$ are expressed in terms of $n_{\mathrm{S}}$ generalized coordinates $c_{j}(t)$ :

$w_{Y}(x, t)=\sum_{j=1}^{n_{\mathrm{S}}} \boldsymbol{v}_{j, \gamma}(x) c_{j}(t), \quad \gamma=1,2, \ldots, J$.

The form functions $v$ are given by the structural models.

The fluid contains $n_{F}$ internal degrees of freedom which, in FLUX, are the discrete pressure values $p_{p}$ in the different mesh cells. The total vector of generalized coordinates $\boldsymbol{d}$ comprises the structural components $c=$ $\left\{c_{j}\right\}$ and fluid parameters $\boldsymbol{p}=\left\{p_{\ell}\right\}: \boldsymbol{d}^{\mathrm{T}}=\left(\boldsymbol{c}^{\mathrm{T}}, \boldsymbol{p}^{\mathrm{T}}\right)$. (Superscript $T$ denotes transposed vectors or matrices.)

In the previous version "FLUX2" of the code, i.e. without active supports with clearances, the coupled fluid-structure system satisfies the equation of motion in the form

$M \ddot{d}+D \dot{d}+S \boldsymbol{d}=r_{1}(t)+r_{2}(t)$.

Here $\boldsymbol{M}, \boldsymbol{D}$ and $\boldsymbol{S}$ correspond to the mass, damping, and stiffness matrices, respectively. They are positive definite or semi-definite and can be transformed into symmetric matrices. The forcing includes the "linear" forces $\boldsymbol{r}_{1}$ due to imposed forces and pressure boundary values and the "nonlinear" forces $r_{2}$. The latter is the contribution from nonlinear fluid convection, $\boldsymbol{r}_{2}=\boldsymbol{r}_{2}(\boldsymbol{d}, t)$. For the further discussion we assume that these quantities are defined as in the previous code version.

\section{The fluid-structure model with impacts}

The above model has to be generalized to account for finite gaps and support forces. Using eq. (8), we can express the vector of gaps $g:=\left\{g_{i}\right\}$, with $g_{i}$ as defined in eq. (1), in terms of the initial gaps $s:=\left\{s_{i}\right\}$ and the generalized coordinates $\boldsymbol{d}$ as

$$
\begin{aligned}
\boldsymbol{g}= & \boldsymbol{s}-\boldsymbol{V} \boldsymbol{d}, \quad \boldsymbol{V}=\left[V_{i, j}\right], \\
V_{i, j}= & \boldsymbol{e}_{i} \cdot\left[\boldsymbol{v}_{j, \boldsymbol{x}_{i}}\left(\boldsymbol{x}_{i}\right)-\boldsymbol{v}_{j, \beta_{i}}\left(\boldsymbol{x}_{i}\right)\right] \\
& i=1,2, \ldots, m: \quad j=1,2, \ldots, n_{\mathrm{S}}, \\
\boldsymbol{V}_{i, j}= & 0 \quad \text { for } j=n_{\mathrm{S}}+1, \ldots, n_{\mathrm{S}}+n_{\mathrm{F}} .
\end{aligned}
$$

As a consequence of the principle of virtual work, the support forces $\boldsymbol{k}:=\left\{k_{i}\right\}$ result in generalized forces $V^{\mathrm{T}} \boldsymbol{k}$ so that the equations of motion instead of eq. (9) now read as

$\boldsymbol{M} \ddot{\boldsymbol{d}}+\boldsymbol{D} \dot{d}+S \boldsymbol{d}=\boldsymbol{r}_{1}+\boldsymbol{r}_{2}+\boldsymbol{V}^{\mathrm{T}} \boldsymbol{k}$.

In order to close the system, we have to reformulate eqs. (5) and (6) for the forces in matrix notation. These equations are formulated as alternatives depending on the state vector $\theta:=\left\{\theta_{i}\right\}$. In order to summarize these conditional equations we define an "activity-matrix" $A$ and a "passivity-matrix" $\boldsymbol{P}$ : Let $\boldsymbol{A}$ be the $\boldsymbol{m}^{\prime} \times m$ matrix which results from a $m \times m$ unit matrix be deletion of those rows $i$ for which $\theta_{i}=0$. Let $P$ be the $\left(m-m^{\prime}\right) \times m$ matrix which results from the $m \times m$ unit matrix by deletion of those rows $i$ for which $\theta_{i}=1 . A$ and $P$ are like $\theta$ motion dependent.

For example, let $m=6, \boldsymbol{\theta}^{\mathrm{T}}=(0,1,0,0,0,1)$. Then, $m^{\prime}=2$ and

$A=\left[\begin{array}{l}010000 \\ 000001\end{array}\right], \quad \boldsymbol{P}=\left[\begin{array}{l}100000 \\ 001000 \\ 000100 \\ 000010\end{array}\right]$.

The matrices are defined such that $\boldsymbol{A g}$ are the gaps of the active supports and $\boldsymbol{P g}$ those of the passive ones. It is easy to verify that

$\boldsymbol{P A}^{\mathrm{T}} \boldsymbol{x}=\mathbf{0}$,

i.e. equal to a null vector $\boldsymbol{\theta}$, for any $m^{\prime}$-component vector $\boldsymbol{x}$.

With these notations, eqs. (5) and (6) imply

$A F k=A g$,

$P k=P O$

Here, $\boldsymbol{F}=\operatorname{diag}\left(F_{1}, F_{2}, \ldots, F_{m}\right)$ is the $m \times m$ diagonal matrix which contains the flexibilities $F_{i}$. Together with initial conditions $d(0)$ and $\dot{d}(0)$, eqs. $(10,11,14,15)$ form a complete system of equations for the unknowns $d(t)$ and $k(t)$. [The initial values for $k(0)$ follow from $d(0)$ and eqs. $(14,15)$.] However, it contains stepwise chang- 
ing matrices $\boldsymbol{A}$ and $\boldsymbol{P}$. A mathematical analysis of the uniqueness of the solution of such a system is not available. From physical reasoning an additional condition appears necessary which requires that for elastic impacts the sum of kinetic and potential energy be continous at the times of impacts where any $\theta_{i}$ changes its value.

\section{Time discretization, Newmark- $\beta$-scheme}

For discretization in time we use the implicit Newmark- $\beta$-scheme [12]. The time axis is discretized in finite time steps of size $\Delta t$. The approximate solutions at times $t_{n}=n \Delta t$ are denoted with indices $n$. Then the discrete analogon to eq. (11) is

$$
\begin{aligned}
& M^{\prime}\left(d_{n+1}-2 d_{n}+d_{n-1}\right)+D^{\prime}\left(d_{n+1}-d_{n-1}\right) \\
& +\boldsymbol{S}\left[\boldsymbol{\beta d _ { n + 1 }}+(1-2 \beta) \boldsymbol{d}_{n}+\beta \boldsymbol{d}_{n-1}\right] \\
& =\beta \boldsymbol{r}_{1}\left(t_{n+1}\right)+(1-2 \beta) \boldsymbol{r}_{1}\left(t_{n}\right)+\beta \boldsymbol{r}_{1}\left(t_{n-1}\right)+\boldsymbol{r}_{2}\left(t_{n}\right) \\
& +\boldsymbol{V}^{\mathrm{T}}\left[\beta \boldsymbol{k}_{n+1}+(1-2 \beta) \boldsymbol{k}_{n}+\beta \boldsymbol{k}_{n-1}\right] \text {. }
\end{aligned}
$$

Here $\boldsymbol{M}^{\prime}:=\boldsymbol{M} / \Delta t^{2}, \boldsymbol{D}^{\prime}=\boldsymbol{D} /(2 \Delta t)$. Note that the nonlinear forces $r_{2}$ are treated explicitly. Further, $\boldsymbol{\theta}_{n+1}$ is determined according

$\boldsymbol{g}_{n+1}=s-\boldsymbol{V} \boldsymbol{d}_{n+1}\left\{\begin{array}{l}>0 \rightarrow \theta_{i}=0 \\ \leqslant 0 \rightarrow \theta_{i}=1\end{array}\right.$,

The matrices $\boldsymbol{A}_{n+1}, \boldsymbol{P}_{n+1}$ are constructed from $\boldsymbol{\theta}_{n+1}$ as described above. The support forces satisfy eqs. $(14,15)$, i.e.

$A_{n+1} F k_{n+1}=A_{n+1} g_{n+1}$,

$\boldsymbol{P}_{n+1} \boldsymbol{k}_{n+1}=\boldsymbol{P}_{n+1} \boldsymbol{O}$.

From initial values we know $\boldsymbol{d}_{0}, \boldsymbol{d}_{-1}$. The unknowns $d_{n+1}, n=0,1, \ldots$, satisfy eq. (16) which in abbreviated form reads

$$
B d_{n+1}=\boldsymbol{r}_{n}^{\prime}+\beta \boldsymbol{V}^{\mathrm{T}} \boldsymbol{k}_{n+1} \text {, }
$$

where

$$
\begin{aligned}
\boldsymbol{B}:= & \boldsymbol{M}^{\prime}+\boldsymbol{D}^{\prime}+\beta \boldsymbol{S}, \\
\boldsymbol{r}_{n}^{\prime}:= & \boldsymbol{\beta} \boldsymbol{r}_{1}\left(t_{n+1}\right)+(1-2 \beta) r_{1}\left(t_{n}\right)+\beta r_{1}\left(t_{n-1}\right) \\
& +\boldsymbol{r}_{2}\left(t_{n}\right)+\boldsymbol{V}^{\mathrm{T}}\left[\beta \boldsymbol{k}_{n-1}+(1-2 \beta) \boldsymbol{k}_{n}\right] \\
& +[2 \boldsymbol{M}-(1-2 \beta) \boldsymbol{S}] \boldsymbol{d}_{n}-\left[\boldsymbol{M}+\boldsymbol{\beta S}-\boldsymbol{D}^{\prime}\right] \boldsymbol{d}_{n-1} .
\end{aligned}
$$

We note that we can rely on $\operatorname{det}(B) \neq 0$ even in cases of singular stiffness matrices $S$ because of the inertia and damping contributions. This point is relevant, e.g., if the core barrel is connected to the vessel by supports with clearances only in a way that the static deflection is non-unique.

For cases without impacts the Newmark- $\beta$-scheme is known to be second order accurate, free of numerical damping, and linearily unconditionally stable for $\beta \geqslant$ $1 / 4$. In the past $\beta=1 / 4$ was the most common choice. We will see that with impacts $\beta=1 / 2$ is preferable although this implies a somewhat larger truncation error (still second-order but of larger size). In the actual code we introduce $\delta d_{n+1 / 2}:=d_{n+1}-d_{n}$ as the actual unknown instead of $\boldsymbol{d}_{n+1}$ hoping that this quantity is less susceptible to numerical round-off errors. However, the write-up is shorter in terms of the above set of variables.

\section{Solution algorithm}

The essential remaining task is to set up an efficient solution scheme to compute the unknowns $\boldsymbol{d}_{n+1}$ and $\boldsymbol{k}_{n+1}$ at each time step, $n=0,1,2, \ldots$ (in this section the time indices will be omitted for shortness).

The existing code contains an efficient solution scheme to solve repeatedly systems $\boldsymbol{B x}=\boldsymbol{y}$ for a set of given right-hand-sides $y$. The efficiency of this solution scheme is based on the use of fast Fourier transform and fast elliptic solvers [5]. These special algorithms are tailored to the structure of $\boldsymbol{B}$ and would be no longer applicable if one would try to solve the extended system (16-19) directly. Therefore, we are looking for a solution scheme which uses the already existing one. For linear systems such a technique is known as capacitance [8] or influence matrix technique [5]. This technique requires precomputation of an influence matrix $E$. In the linear case, $\boldsymbol{E}$ is a constant. In the present nonlinear case we will define a solution dependent influence matrix $E^{\prime}$. The essential point is, however, that $E^{\prime}$ can be constructed easily from a single precomputed matrix $\boldsymbol{E}$. As we will recognize later, the matrix $\boldsymbol{E}$ is defined by

$\boldsymbol{E}=\boldsymbol{V} \boldsymbol{B}^{-1} \boldsymbol{V}^{\mathrm{T}}$.

It is a $m \times m$ matrix. As $V^{\mathrm{T}}$ contains $m$ columns, construction of $\boldsymbol{E}$ requires $m$ solutions with the existing scheme which solves $\boldsymbol{B} \boldsymbol{x}=\boldsymbol{y}$. This effort is small in comparison to the overall effort because $m$ is usually much smaller than the number of time steps. The meaning of $\boldsymbol{E}=\left[E_{i, j}\right]$ is such that $E_{i, j}$ is the displacement of the fluid-structure system for passive supports during one time-step at support position $i$ due to a unit force at position $j$. 
Then, at each time step, we obtain the solution $\boldsymbol{d} \equiv \boldsymbol{d}_{n+1}, \boldsymbol{k} \equiv \boldsymbol{k}_{n+1}$ in the following steps:

(1) Set

$$
\bar{k}=0 \text {, }
$$

and determine the solution which would be the final one if all supports are passive after this time step.

$\boldsymbol{B} \bar{d}=\boldsymbol{r}^{\prime}$.

The related actual gaps are

$\widetilde{g}=s-V \tilde{d}$.

According to $\overline{\boldsymbol{g}}$ we determine the impact state vector $\boldsymbol{\theta}$ as in eq. (17) and the number $\bar{m}^{\prime}$ of active supports as in eq. (4).

(2) If $\bar{m}^{\prime}=0$, then no support is active and we have the required solution, i.e.

$\boldsymbol{d}=\overline{\boldsymbol{d}}, \quad \boldsymbol{k}=\overline{\boldsymbol{k}}$.

(3) Otherwise, we have $\bar{m}^{\prime}>0$ active supports. Then, we proceed and set up $\boldsymbol{A}$ and $\boldsymbol{P}$ according to $\boldsymbol{\theta}$. From this we construct the actual influence matrix

$\boldsymbol{E}^{\prime}=\boldsymbol{A}(\boldsymbol{\beta} \boldsymbol{E}+\boldsymbol{F}) \boldsymbol{A}^{\top}, \quad \operatorname{det}\left(\boldsymbol{E}^{\prime}\right) \neq 0$.

As will be proven later, the impact forces are

$\boldsymbol{k}=\boldsymbol{A}^{\mathrm{T}}\left(\boldsymbol{E}^{\prime}\right)^{-1} \boldsymbol{A} \overline{\boldsymbol{g}}$.

(4) We now check whether all forces are non-positive. If for any $i, k_{i}>0$, then we set $\theta_{i}=0, \bar{m}^{\prime}:=\bar{m}^{\prime}-1$, and repeat from step (3). The reason for this loop will be explained later in this chapter.

(5) With known forces $\boldsymbol{k}$ we solve the $\boldsymbol{B}$-system the second time, i.e. determine $d$ from

$\boldsymbol{B d}=\boldsymbol{r}^{\prime}+\beta \boldsymbol{V}^{\mathrm{T}} \boldsymbol{k}$.

With this solution the new gaps are

$g=s-V d$,

and, according to the sign of $g_{i}$ we have a new state vector $\theta^{*}$ :

$\theta_{i}^{*}=1$ if $g_{i} \leqslant 0$ or $\bar{\theta}_{i}=1$

(The "or" condition is used to avoid infinite loops which can appear due to round-off errors which indicate small positive gaps.)

(6) Now it can happen, that the added impact forces have increased the number of closed gaps so that not all $\theta_{i}^{*}=\bar{\theta}_{i}$.

In this case we set $\theta:=\theta^{*}$ and $\bar{m}^{\prime}:=\sum_{i=1}^{m} \theta_{i}^{*}$ and repeat with step (3). Otherwise the solution is found.
Now, we have to prove that eq. (30) indeed gives the correct forces which satisfy eqs, (18) and (19), i.e.

$A F k=A g$,

$\boldsymbol{P k}=\boldsymbol{P O}$.

Eq. (35) is satisfied because of eq. (13) and eq. (30). The proof of eq. (34) is obtained by successively inserting the defining equations. The bracketed numbers above equality signs denote the number of equation used:

$$
\begin{aligned}
& A \boldsymbol{F} \boldsymbol{k}-\boldsymbol{A} \boldsymbol{g} \stackrel{(32)}{=} \quad \boldsymbol{A F k}-\boldsymbol{A s}+\boldsymbol{A} \boldsymbol{V d} \\
& \stackrel{(31)}{=} A F K-A s+A V B^{-1} r^{\prime} \\
& +\beta \boldsymbol{A} \boldsymbol{V B}^{-1} \boldsymbol{V}^{\mathrm{T}} \boldsymbol{k} \\
& \stackrel{(24)}{=} \quad A(\boldsymbol{F}+\beta E) k-A s+A V B^{-1} \boldsymbol{r}^{\prime} \\
& \stackrel{(30)}{=} \quad A(F+\beta E) A^{\mathrm{T}}\left(E^{\prime}\right)^{-1} A \bar{g} \\
& -\boldsymbol{A} s+\boldsymbol{A} V \boldsymbol{B}^{-1} \boldsymbol{r}^{\prime} \\
& \stackrel{(29)}{=} A\left(\bar{g}-s+V B^{-1} r^{\prime}\right) \\
& \stackrel{(27)}{=} A\left(-V \bar{d}+V B^{-1} \boldsymbol{r}^{\prime}\right) \\
& \stackrel{(26)}{=} \quad A O, \text { q.e.d. }
\end{aligned}
$$

Thus, we can be sure that the above algorithm determines the forces just so that the support constraints are satisfied.

The intermediate step (4) is introduced to avoid positive support forces. A positive support force contradicts eq. (7) and means that the support is attracting
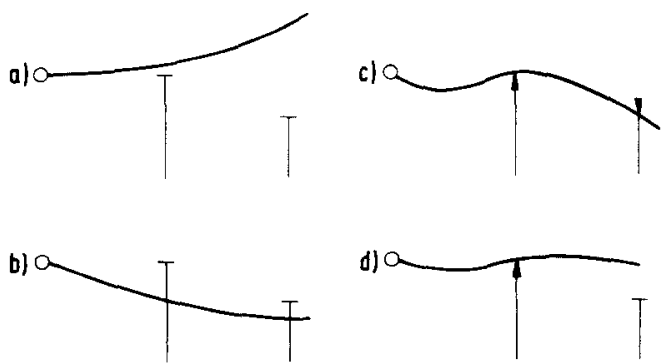

Fig. 4. States in the solution scheme: (a) initial state. two passive supports; (b) state after first solution step indicating two active supports; (c) for one of the enforced supports a positive force appears; (d) final state with one active support imposing negative force. 
instead of deflecting the structure. Fig. 4 illustrates the different stages on the solution scheme and shows that such false forces can appear. Since the amount of computing work envolved in steps (3) and (4) is small, this possibility does not deteriorate the efficiency of the solution scheme. The number of iterations of (3) and (4) is finite because the number of active supports is reduced each time and cannot go below one. Whereas the check in step (4) implies a reduction in the number of active supports, the check in step (6) enlarges this number. Again, the maximum number of supports is finite so that this loop too cannot cause infinite loops.
The only possibility conceivable for infinite loops comes from a combination of the checks in steps (4) and (6). This case did never appear in the practical applications. To make the algorithm definitely finite, the maximum number of iterations is limited to $m+1$ in the code. It should be noted that for all $2^{m}$ possible support configurations, $2^{m}$ matrices $\boldsymbol{E}^{\prime}$ arise. One can think of storing all these matrices. However, in the actual code it is simpler and not time consuming to reconstruct $\boldsymbol{E}^{\prime}$, according to its definition from $\boldsymbol{E}$ and $\boldsymbol{F}$. This allows, moreover, to make $\boldsymbol{F}$ dependend on the solution at time $t_{n}$ so that weakly nonlinear flexible supports can easily
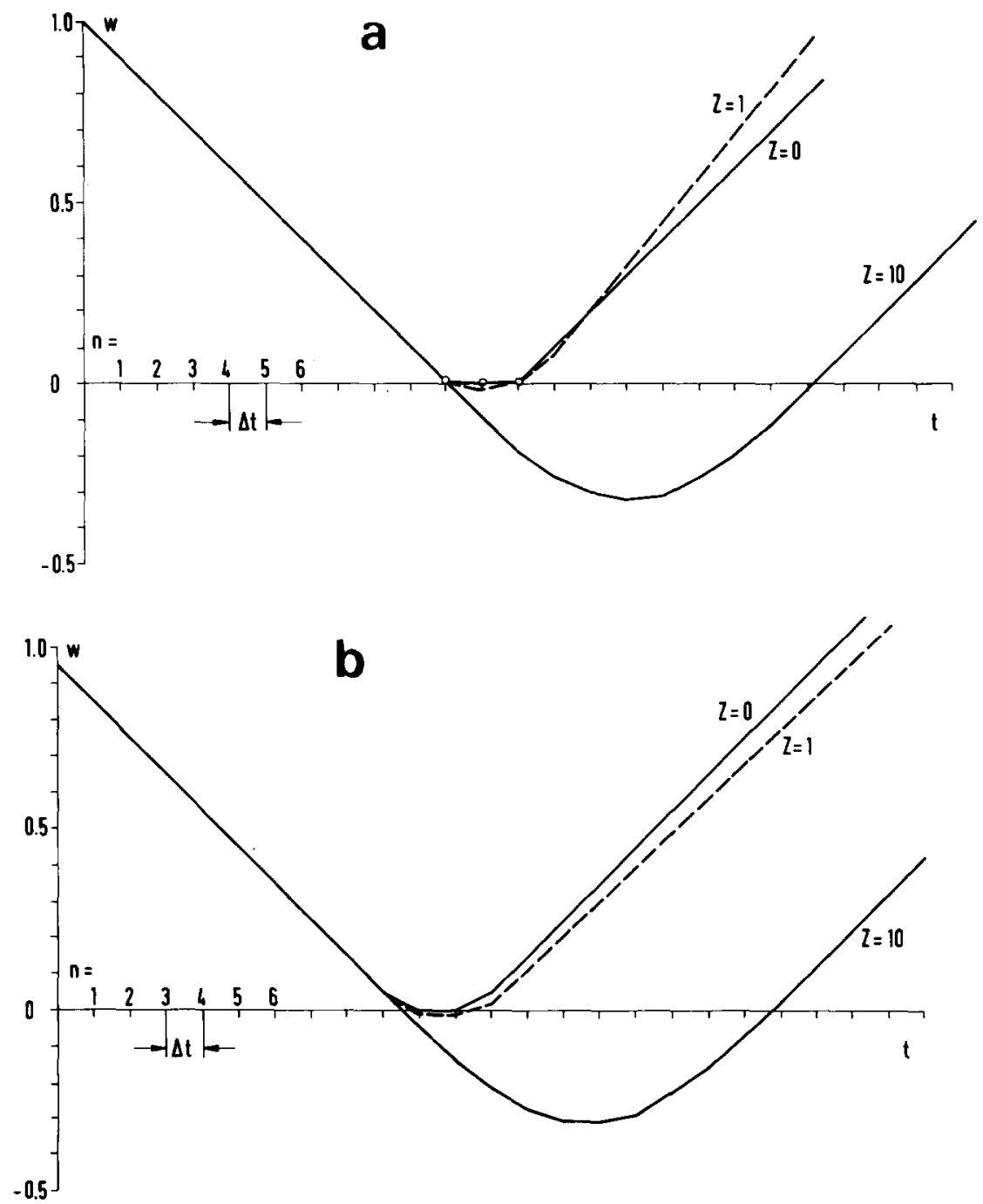

Fig. 5. Motion $w(t)$ of a mass-point, (a) hitting the support at $w=0$ at the end of a time interval, and (b) hitting the support in the mid-point of a time interval, for different support flexibilities $F, Z=T / \Delta t, T=\pi \sqrt{M F}$, using the Newmark- $\beta$-scheme with $\beta=1 / 2$. 
be included. By this means plastic supports can be simulated.

\section{Tests with a one-degree-of-freedom system}

Before a new algorithm is ready for implementation in a complex program it should be tested for simple cases with analytical solutions. The most simple case seems to be the one-mass-system

$M \ddot{u}=k$.

$k=0$ for $w>0$,

$F k=w$ for $w<0, \quad F \geqslant 0$,

$\dot{w}(0)=-1, \quad w(0)=w_{0}>0$.

This problem corresponds to that of a ball bouncing against a wall. For elastic supports $(F>0)$ the velocity after impact should be negative the initial value. The duration of contact with the support is

$T=\pi \sqrt{M F}$.

Fig. 5 shows the numerical result obtained for the model problem using the above algorithm with $\beta=1 / 2$ for different values of $F$ or - what is the relevant non-dimensional parameter - values of $Z=T / \Delta t$. For rigid supports, $F=0$, so that $Z=0$. It is not surprising that in the numerical scheme the impact time is finite and of the order $\Delta t$. Thus, the numerical scheme with impacts is only first-order accurate.

More disturbing is the fact that the magnitude of the velocity changes. As we can see from fig. 5 , the scheme is not energy conserving for flexible supports. The energy change can be both positive and negative. The largest energy change appears if the theoretical impact time when the mass hits the support is exactly $t^{\prime}=t_{n}$ (fig. 5a). The energy change is a minimum if $t^{\prime}=(n+$ 1/2) $\Delta t$ (fig. $5 b$ ). The dependence of the energy change on $Z$ is shown in fig. 6 . It is less than $1 \%$ for $Z \leqslant 10^{-2}$ and for $Z \geqslant 10^{2}$. For intermediate values of $Z$ the energy change can be as large as about $50 \%$. But we can expect that the relative energy change will be much smaller in a system with many degrees of freedom and with potential energy in addition to kinetic energy. Nevertheless, the algorithm exhibits numerical energy dissipation (or production) as is often found in first order schemes [2]. However in the present scheme energy changes will appear only for the finite number of time instances where impacts occur. The above algorithm should be applied, therefore, either for rigid supports $(Z=0)$ or for very flexible supports where the

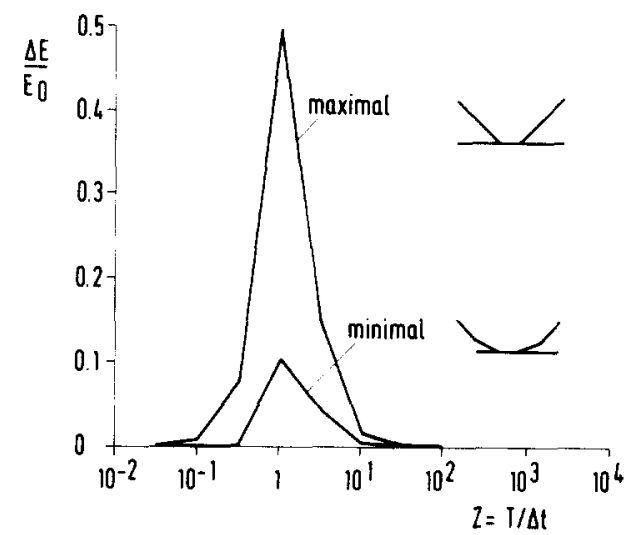

Fig. 6. Relative energy change during impact in the Newmark$\beta$-scheme with $\beta=1 / 2$ in the worst and best case as a function of the ratio of theoretical impact time $T$ over time step $\lrcorner t$ as found for the one-degrees-of-freedon model.

contact time $T$ is large in comparison to the time step size $\Delta t$.

It is important to note that these results have been obtained for a Newmark-parameter $\beta=1 / 2$. For $\beta \neq$ $1 / 2$, the energy change is usually larger and non-zero also for $Z=0$. Thus, the value $\beta=1 / 2$ should be used always in this method.

\section{Energy conserving integration scheme}

The above experience with a one-degree-of-freedom model calls for a deeper analysis. For this purpose we first show that the Newmark- $\beta$-scheme without impacts indeed conserves a finite difference form of the total energy $T$, i.e. the sum of kinetic and potential energy. (For a similar discussion see [13].)

We introduce difference and average operators which, for any quantity $a(t)$, are defined by

$$
\begin{aligned}
& \delta a(t) \equiv a(t+\Delta t / 2)-a(t-\Delta t / 2), \\
& \bar{a}(t) \equiv[a(t+\Delta t / 2)+a(t-\Delta t / 2)] / 2 .
\end{aligned}
$$

One can easily verify the following identities

$\delta(a b)=\bar{a} \delta b+\bar{b} \delta a$,

$\overline{a b}=\bar{a} \bar{b}+\delta a \delta b / 4$.

We claim that the Newmark- $\beta$-scheme without impacts conserves

$T^{\prime}:=\left(\delta d^{\top} M^{\prime} \delta d+\bar{d}^{\mathrm{T}} \boldsymbol{S} \bar{d}\right) / 2$, 
and the change in energy per time step is

$\delta T^{\prime}=-\delta \bar{d}^{\mathrm{T}} D^{\prime} \delta \bar{d}+\delta \bar{d}^{\mathrm{T}} \overline{\overline{\boldsymbol{r}}}$.

The right-hand-side is obviously the work done by damping and external forces per time step. We now have to assume symmetric matrices $\boldsymbol{M}$ and $\boldsymbol{S}$. Then, by means of eq. (41), we can expand eq. (44) with the result

$\delta \bar{d}^{\mathrm{T}}\left(M^{\prime} \delta \delta d+D^{\prime} \delta \bar{d}+S \overline{\bar{d}}-\overline{\bar{r}}\right)=0$

We see that for arbitrary "velocity" $\delta \bar{d}$ this expression is zero if and only if the bracket vanishes. One easily verifies that this gives us just the Newmark-scheme for $\beta=1 / 4$, because

$\overline{\bar{d}}(t)=\frac{1}{4} d(t-\Delta t)+\frac{1}{2} d(t)+\frac{1}{4} d(t+\Delta t)$,

compare eq. (16)! Similarily, one finds that the alternative finite difference form of the total energy

$T^{\prime \prime}:=\left(\delta d^{\mathrm{T}} \boldsymbol{M}^{\prime} \delta d+\overline{d^{\mathrm{T}} S d}\right) / 2$

is exactly conserved by the Newmark-scheme if $\beta=1 / 2$. If we now include the potential energy stored in the support springs, then we have to conserve, e.g.,

$\tilde{\boldsymbol{T}}^{\prime}=T^{\prime}+\frac{1}{2} \overline{\boldsymbol{b}}^{\mathrm{T}} \boldsymbol{F}^{-1} \overline{\boldsymbol{b}}$,

with $b=\min (\boldsymbol{0}, \boldsymbol{s}-\boldsymbol{V d})$. Now

$\delta \tilde{T}^{\prime}=\delta \bar{d}^{\mathrm{T}}\left(M^{\prime} \delta \delta d+S \overline{\bar{d}}\right)+\delta \bar{b}^{\mathrm{T}} F^{-1} \overline{\bar{b}}$

If the time step includes the impact time then $\delta \boldsymbol{d}$ and $\delta \boldsymbol{b}$ are not linearily related. Thus the new solution which satisfies $\delta \tilde{T}^{\prime}=0$ (inclusion of external forces, damping and impact energy losses would be possible) is the solution of a quadratic vector equation.

Thus we have learned that complete energy conservation cannot be obtained with a non-iterative scheme. Instead, we propose to use the above algorithm inspite of not being completely energy conserving. At least we can expect that the proposed algorithm is sufficiently energy conserving with $\beta=1 / 2$ for rigid or very flexible supports. If one wants to know the motion with moderately flexible supports then one has to take small time steps so that $Z \gg 1$. Of course one should expect that other schemes which have been used in the past for integration of multi-degrees-of-freedom systems with impacts show numerical energy changes as well, however no relevant discussion has been found in the literature.

\section{Result of example code applications}

\subsection{Case specification}

The method described above has been implemented in the new code version "FLUX3" and applied for a study of algorithmic performance and of the basic motion behaviour. For this purpose a configuration with parameters which are typical for a PWR has been considered. The complete list of input parameters is documented in an internal report. Here we discuss a prototype calculation so that details should not matter.

Six cases, $\mathrm{C} 0$ to $\mathrm{C} 5$, have been evaluated. The cases differ in the support and fluid-structure interaction parameters as summarized in table 1 . Case $\mathrm{C} 0$ is the FLUX2 reference case with rigid core-barrel flangeclamping and no snubbers. Cases $\mathrm{Cl}$ to $\mathrm{C} 5$ include up to six supports with clearances. Here $\mathrm{Cl}$ is the new FLUX3 reference case with supports as shown in figs. 1 and 2 and parameters as given in table 2 . In case C2 all supports are rigid. C3 is a decoupled analysis; here the fluid pressure is imposed on the structure but the feedback of the structural motion on the fluid is neglected; this feedback is taken into account in all other cases. Cases $\mathrm{C} 1$ to $\mathrm{C} 3$ include a hold-down force at the upper flange which is approximately three times the core barrel weight (including the mass ring which simulates the core mass). In reality, hold-down forces of this order are imposed by the weight and prestressed springs at the upper side of the flange. In case $\mathrm{C} 4$, this force is set to zero in order to show its effect. Finally, in case C5, the upper flange is again rigidly clamped but snubbers are present at the lower edge as in case $\mathrm{Cl}$.

The core barrel is modeled with CYLDY2 as mentioned in section 3 . In order to allow for axial flange

Table 1

Case specification

\begin{tabular}{ll}
\hline Case & Description \\
\hline C0 & $\begin{array}{l}\text { Case without impacts (FLUX2), i.e. rigid clamping } \\
\text { at upper flange, free motion at lower core barrel } \\
\text { edge: including fluid-structure interaction. }\end{array}$ \\
C1 & $\begin{array}{l}\text { Reference case with six supports as defined in table } 2 \\
\text { (FLUX3) and with fluid-structure interaction. }\end{array}$ \\
C2 & As C1 but all supports rigid $\left(F_{i}=0, i=1,2, \ldots, 6\right)$. \\
C3 & As C1 but without fluid-structure coupling. \\
C4 & As C1 but with zero hold-down forces. \\
C5 & As C1 but with rigid clamping at upper flange \\
& $\left(s_{2}=s_{3}=s_{5}=s_{6}=0\right)$.
\end{tabular}


Table 2

Support parameters for Case $\mathrm{C} 1$

\begin{tabular}{lllllll}
\hline $\begin{array}{l}\text { Support No. } \\
\text { (refer to fig. 1) }\end{array}$ & 1 & 2 & 3 & 4 & 5 & 6 \\
\hline $\begin{array}{c}\text { Initial gap } s \\
(\mathrm{~mm})\end{array}$ & 0.5 & 0 & 0.3 & 0.5 & 0 & 0.3 \\
$\begin{array}{c}\text { Flexibility } F \\
\left(10^{-12} \mathrm{~m} / \mathrm{N}\right)\end{array}$ & 10.0 & 0 & 1.0 & 10.0 & 0 & 1.0 \\
$\begin{array}{c}\text { Direction } \boldsymbol{c} \\
(\mathrm{radial} / \mathrm{axial}) \\
\begin{array}{c}\text { Hold-down force } \\
(\mathrm{MN})\end{array}\end{array}$ & $\mathrm{r}$ & $\mathrm{a}$ & $\mathrm{a}$ & $\mathrm{r}$ & $\mathrm{a}$ & $\mathrm{a}$ \\
\hline
\end{tabular}

motion, rigid body modes have been added, one for the zeroth and one for the first cosine mode (corresponding to uniform axial or turning motion). At the flange, realistic springs and inertia parameters are included. The resultant dynamic properties can be characterized by the computed basic eigenfrequencies in vacuum. For rigid flange clamping (cases $\mathrm{C} 0$ and $\mathrm{C5}$ ) the lowest eigenfrequencies are $353 \mathrm{~Hz}$ in the zeroth and $17.9 \mathrm{~Hz}$ in the first cosine mode. In cases $\mathrm{Cl}$ to $\mathrm{C} 4$ the corresponding lowest frequencies are $25 \mathrm{~Hz}$ (axial) and 5.6 $\mathrm{Hz}$ (beam mode), respectively.

In all cases, a total of $n_{\mathrm{S}}=213$ structural degrees of freedom and $n_{\mathrm{F}}=4975$ discrete pressure values has been included. A time period of $40 \mathrm{~ms}$ is covered with 200 time steps $(\Delta t=0.2 \mathrm{~ms}$ ). In this period the fluid inside the pressure vessel remains in its liquid state (subcooled). At the break the pressure is prescribed to drop from its initial value of $16 \mathrm{MPa}$ within $5 \mathrm{~ms}$ following a smooth ramp to the saturation pressure of $9.2 \mathrm{MPa}$.

\subsection{Description of the computed motions}

Fig. 7 indicates the time periods of activity of the different supports with clearances. Simplest to interpret is case $\mathrm{C} 5$ with rigid clamping at the upper flange. As expected, the core barrel hits first the snubber on the blowdown side. The contact duration is between one and three time steps for rigid supports and of the order of 25 to 50 time steps for flexible snubbers if the fluid inertia is taken into account. These contact times are large enough to suggest that numerical damping is
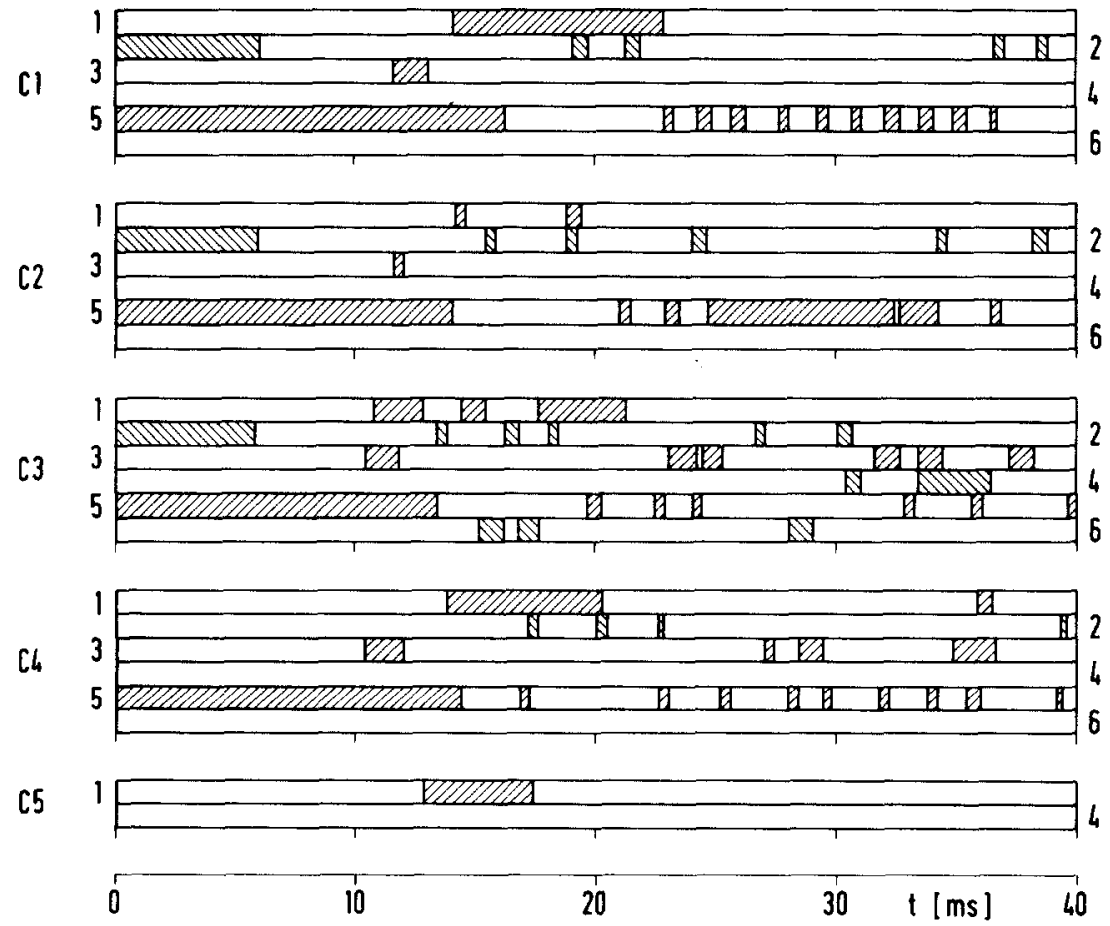

Fig. 7. Support state diagram for cases $\mathrm{Cl}$ to $\mathrm{C} 5$. The shaded bands indicate periods of activity of the support with corresponding number. 
negligible. In the time period considered, the second snubber (support 4) is hit only if the fluid-structure interaction is not included (case C3). Of course, these results depend strongly on the assumed flexibilities and initial gaps. In reality, the flexibility and finite inertia of the pressure vessel wall and plastic deformation of the sunbbers will be important for such dynamic loadings.

Fig. 8 shows the radial deflection of the core barrel at support 1 . We see that the snubbers effectively limit the motion amplitude. Flexible snubbers allow, of course, some deflection beyond the initial clearances. Obviously, large accelerations arise. It might be interesting to study the forces implied by these accelerations on the core internals. We should note, that the present results are obtained for a structural model which assumes a rigid mass ring connected to the lower core barrel edge. In reality this part will deform elastically.

Case $\mathrm{C} 3$ without fluid-structure interactions shows large motion amplitudes and larger frequencies at this and other points. In fact, fluid-structure interaction is still the dominant physical effect for the global motions. This can be concluded in particular from fig. 9 which shows the radial core barrel deflection near the blowdown nozzle. The differences between cases $\mathrm{C} 0$ and $\mathrm{Cl}$ are much smaller than those between $\mathrm{C} 0$ and $\mathrm{C} 3$. The

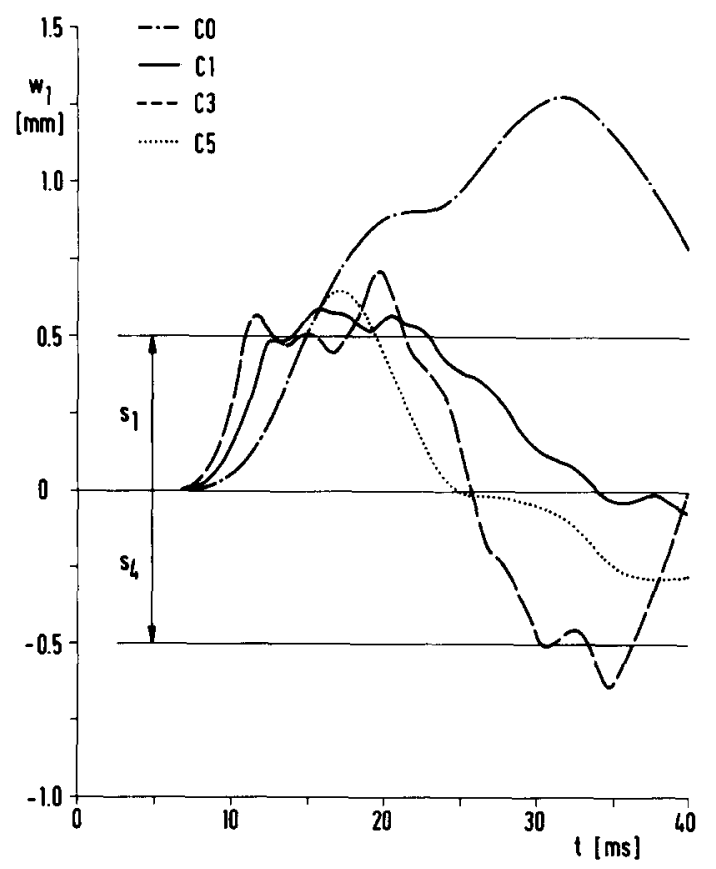

Fig. 8. Radial core barrel deflection at the lower edge near support 1 versus time for cases $\mathrm{C} 0, \mathrm{Cl}, \mathrm{C} 3$ and $\mathrm{C} 5$. Except for case $\mathrm{C} 0$ the snubbers are active for $\left|w_{1}\right| \geqslant 0.5 \mathrm{~mm}$.

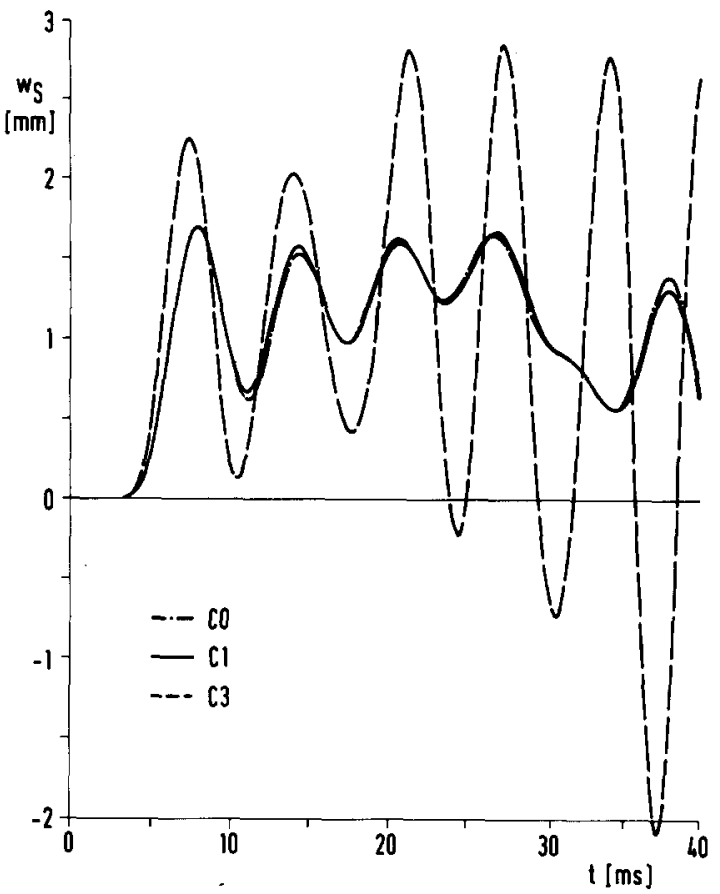

Fig. 9. Radial core barrel deflection near blowdown nozzle versus time for cases $\mathrm{C} 0$ and $\mathrm{Cl}$ (without and with impacts) and $\mathrm{C} 3$ (with impacts but without fluid-structure interaction).

integral horizontal pressure loading amplitudes on the core barrel, see fig. 10, are reduced in the case with impacts but slightly.

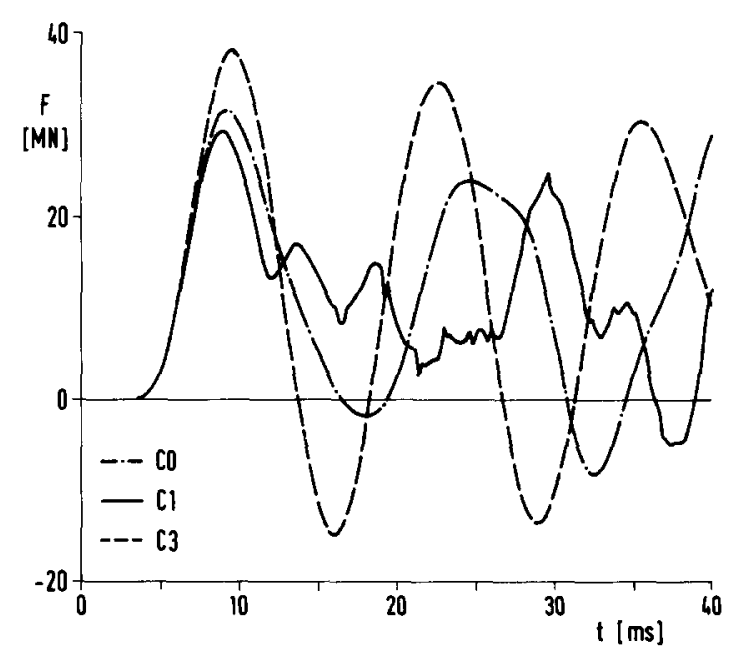

Fig. 10. Integral pressure loading force on the core barrel in the direction of the blowdown pipe versus time for cases $\mathrm{CO}, \mathrm{Cl}$ and $\mathrm{C} 3$. 


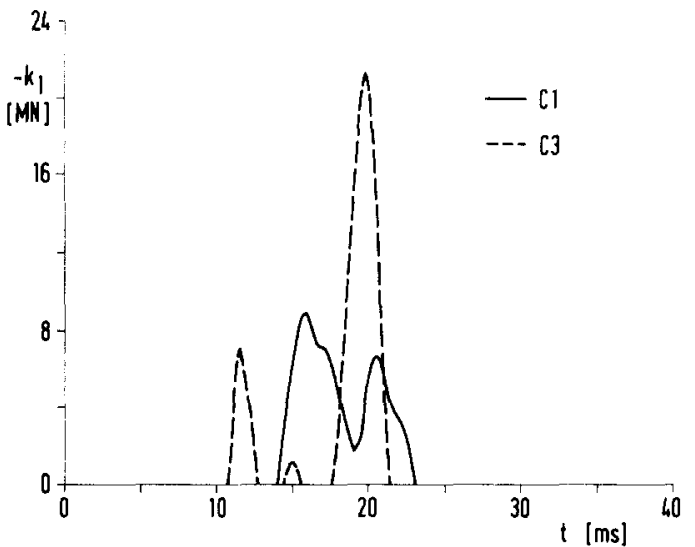

Fig. 11. Impact force magnitude at the snubber (support 1) versus time with $(\mathrm{Cl})$ and without $(\mathrm{C} 3)$ fluid-structure interaction.

Also, the impact forces, shown for example for the snubber in fig. 11, are more than two times larger if the fluid-structure interaction is neglected. This fact was not clear a priori because the added fluid mass has two opposing effects: it enlarges the inertia but reduces the velocity at impact. Such information is certainly of large importance for proper layout of the snubbers.

Very complex motions arise if the core barrel flange is supported with clearances. This can be seen from fig. 7 and in more detail for case $\mathrm{Cl}$ from fig. 12. The pressure load imposes a moment to the flange with a maximum of $85 \mathrm{MNm}$. This moment is about eight times larger than the moment of the flange holddown forces so that these forces do not prevent the flange from lifting *. They only delay this motion (see cases $\mathrm{Cl}$ and $\mathrm{C} 4$ in fig. 7). The flange lifts first at the side of the blowdown pipe. At certain times it totally looses the contact at the lower side. Without fluid-structure interaction, C3, the upward motion is large enough so that the flange can hit the upper supports 3 and 6 . With fluid-structure interaction these motion amplitudes are smaller. The assumption of rigid supports 2 and 5 result in interesting bouncing motions, see fig. 12. Again, such an assumption might be unrealistic. However, this result demonstrates the good performance of the algorithm used.

For evaluation of safety margins, one has to consider carefully the strong local stresses which are to be expected near the impacting supports in the core barrel.

* Additional hold-down forces arise in the course of the blowdown due to axial pressure drop along the core.

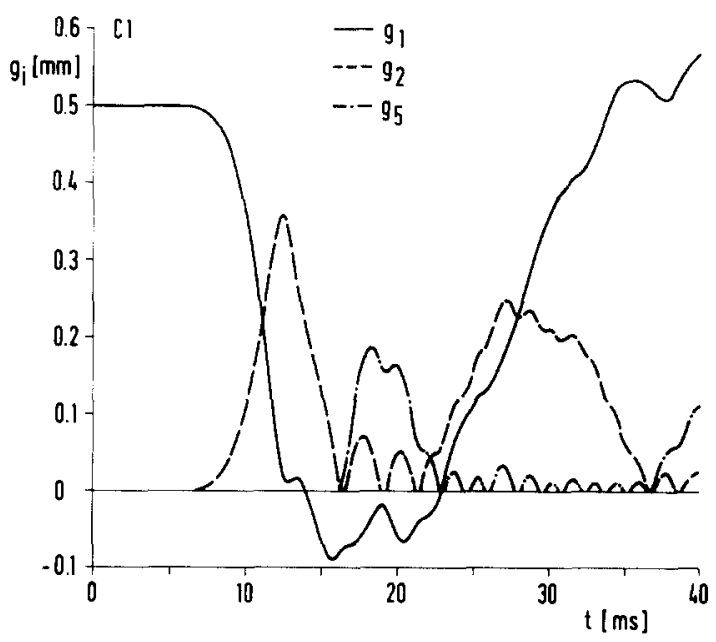

Fig. 12. Gap at supports number 1, 2, and 5 versus time for case $\mathrm{Cl}$.

Our global shell model is not designed for this purpose. Our results show, however, strong shell curvature in the shell after the impact, see fig. 13. In fact, by comparison of cases $\mathrm{C} 0$ and $\mathrm{C} 5$ it has been found that the lower snubbers introduce an increase in the maximum shell bending stresses by about $20 \%$, but a decrease of the maximum membrane stresses by about $3 \%$. The latter are those contributing mainly to the primary stresses. Thus the efficiency of the snubbers in reducing the stresses is small.

\subsection{Numerical performance}

Case $\mathrm{C} 0$, i.e. the situation without impacts, requires a computing time of 22 minutes IBM 370/3033. The maximum computing time with impacts was 33 minutes (see table 3 ). Even if impacts would be active at each time step, the increase in computing time amounts to only a factor of 1.7 and thus less than a factor of two although two solutions of the original linear equations are required every time step. This is due to unchanged parts of the program required for evaluation of the nonlinear fluid convection and other evaluations. The need for (cheap) iterations of the support forces (step 4 of the solution algorithm) arises in typically $5 \%$ of the integration steps (see table 3 ). Very rarely a third solution of the linear equations was necessary due to support forces which caused an additional support to become active (step 6 of the solution algorithm), see table 3. Thus these numbers of iterations are negligible. The extra amount of computer storage for the extended 


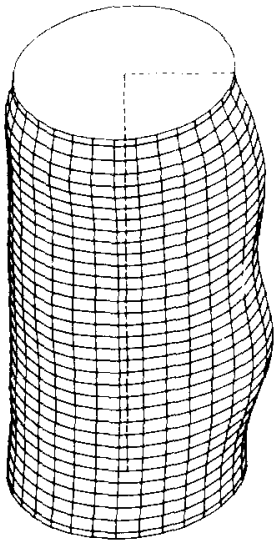

$t=16$

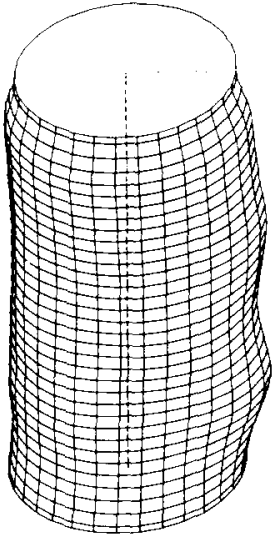

18

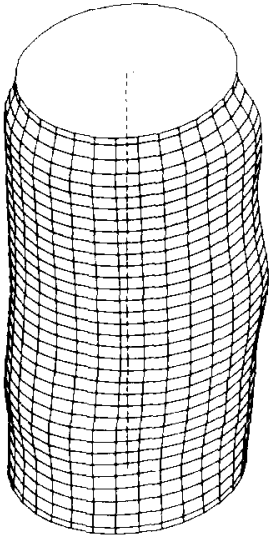

20

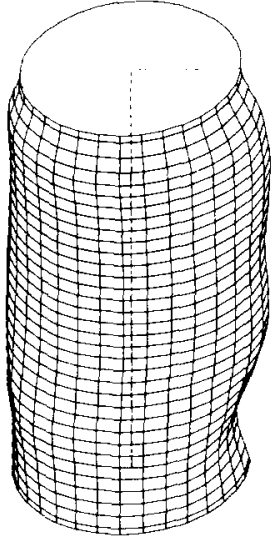

22 [ms]

Fig. 13. Perspective plots of the deformed core barrel for a sequence of times during impact of the snubber at the lower core barrel edge in case $\mathrm{C} 1$. The core barrel deflection is 300 times magnified in order to make the rather small deflections visible. The blowdown pipe and the active snubber are located on the right side in this figure.

Table 3

Performance parameters *

\begin{tabular}{lllll}
\hline Case & $t_{c}$ & $n_{0}$ & $n_{4}$ & $n_{6}$ \\
\hline $\mathrm{CO}$ & 22 & 200 & - & - \\
$\mathrm{Cl}$ & 32 & 347 & 7 & 0 \\
$\mathrm{C} 2$ & 33 & 347 & 11 & 1 \\
$\mathrm{C} 3$ & 33 & 364 & 7 & 1 \\
$\mathrm{C} 4$ & 32 & 340 & 7 & 1 \\
$\mathrm{C} 5$ & 24 & 225 & 0 & 0
\end{tabular}

$* t_{\mathrm{c}}=$ computing time in minutes IBM 3033 ,

$n_{0}=$ number of solutions with old linear solver per 200 time steps,

$n_{4}=$ number of iterations according to step 4,

$n_{6}=$ number of iterations according to step 6 of the solution algorithm (see section 6).

method is less than $5 \%$ and mainly required to keep the matrix $V$ defined in eq. (10).

\section{Conclusions}

For analysis of impacts in a three-dimensional fluid-structure system an algorithm has been developed. The method can easily be implemented in existing implicit fluid-structure integration schemes. It has been incorporated in the new variant "FLUX3" of the exist- ing FLUX code. The algorithm is non-iterative in nature and performs efficiently. The code has been found to be numerically stable and reliable.

The method is based on the influence matrix technique. This technique has been found to be extremely helpful for linear problems in the past [5,8]. It allows to solve a complex problem by two solutions of a simpler problem. Here it is used the first time in a situation which is nonlinear. As yet the code has been applied for linear elastic supports only. However, the method is ready for applications where the support flexibilities depend upon the actual motion field so that plastic supports can be treated.

Special attention is required with respect to energy conservation during impact. No relevant discussion has been found in the literature. The proposed scheme conserves energy for rigid supports. For flexible supports, the relative energy change is small if the number of time steps per impact is large. The numerical method has been applied for a series of prototype computations of coupled fluid-structure motions with impacts in a pressurized water reactor during blowdown. We note that still several important parts like the core structure and the dynamic motion of the vessel have not yet been included in the code. Work is in progress for these aspects. Also, the input data are subject to reinspection. But we have found several qualitative results which are of general validity for a typical PWR.

In particular we may conclude that

- impacts have large effects on the global motion be- 
haviour of the system and cause local accelerations and loadings,

- generally, impacts have smaller importance than fluid-structure interaction effects,

- fluid-structure interactions reduce the impact loadings,

- hold-down forces of realistic magnitude do not prevent the upper core-barrel flange from lifting if the flange is supported with clearances,

- the gain in stress reduction due to snubbers is so small that the use of snubbers is debatable.

The present method seems well suited to be applied for analysis of supports with clearances. In particular the method can be used to provide data to optimize the design of such supports with respect to initial clearance, flexibility and plasticity. In this respect, the efficiency of the code is attractive. The inclusion of fluid-structure interaction effects is obviously very important for realistic results. Thus the present study may contribute to demonstrate or even enhance nuclear reactor safety margins.

\section{Acknowledgement}

The author is grateful to Dr. A. Ludwig and Dr. E.G. Schlechtendahl for several stimulating discussions and suggestions, to Mr. H. Knüppel and Mrs. U. Stutz for support in plotting the results, and to Mrs. H. Jansky for typing the manuscript.

\section{References}

[1] E.G. Schlechtendahl, Status of code-development in the Federal Republic of Germany concerning fluid-structural coupling during reactor transients. Nucl. Safety 20 (1979) 551-563.
[2] T. Belytschko and U. Schumann, Fluid-structure interactions in light water reactor systems. Nucl. Engrg. Des. 60 (1980) 173-195.

[3] R. Krieg, E.G. Schlechtendahl and K.H. Scholl, Design of the HDR-experimental program on blowdown loading and dynamic response of PWR-vessel internals. Nucl. Engrg. Des. 43 (1977) 419-435.

[4] L. Wolf, U. Schumann and K.-H. Scholl, Experimental and analytical results of coupled fluid-structure interactions during blowdown of the HDR-vessel, 6th SMiRTConf., Paris, August 1981, B2/1.

[5] U. Schumann, Fast elliptic solvers and three-dimensional fluid-structure interactions in a pressurized water reactor. J. Comput. Phys. 36 (1980) 93-127.

[6] R.L. Cloud, Structural mechanics applied to pressurized water reactor systems. Nucl. Engrg. Des. 46 (1978) 273302.

[7] G.J. Böhm and J.P. Lafaille, Reactor internals response under a blowdown accident. 4th SMiRT-Conf., San Francisco, August 1977, F1/3.

[8] B.L. Buzbee et al., The direct solution of the discrete Poisson equation on irregular regions, SIAM J. Numer. Anal. 8 (1971) 722-736.

[9] U. Schumann and J. Benner, Homogenized model for fluid-structure interactions in a pressurized water reactor core. 6th SMiRT-Conf., Paris, August 1981, B 5/7.

[10] A. Ludwig and R. Krieg, Dynamic response of a clamped/ring-stiffened circular cylindrical shell under non-axisymmetric loading. Nucl. Engrg. Des. 43 (1977) 437-453.

[11] A. Ludwig and R. Krieg, An analytical quasi-exact method for calculating eigenvibrations of thin circular cylindrical shells. J. Sound Vibr. 74 (1981) 155-174.

[12] N.M. Newmark, A method of computation for structural dynamics, J. Engrg. Div. ASCE 85 (1959) NO. EM3, 67-94.

[13] T. Belytschko and D.F Schoeberle, On the unconditional stability of an implicit algorithm for nonlinear structural dynamics, J. Appl. Mech. (Trans. ASME) 42 (1975) 865869. 